13

\title{
Исследование с применением SK-анализа вольт-амперных характеристик нанокомпозитных полевых эмиттеров на основе графена
}

\author{
(С) Е.О. Попов, А.Г. Колосько, С.В. Филиппов, И.Ю. Смирнов \\ Физико-технический институт им. А.Ф. Иофрфе РАН, \\ 194021 Санкт-Петербург, Россия \\ e-mail: e.popov@mail.ioffe.ru
}

(Поступило в Редакцию 23 июня 2015 г.)

Разработан метод онлайн обработки данных полевого эмиссионного эксперимента для исследования эмиттеров большой площади. Метод основан на анализе SK-диаграммы вольт-амперных характеристик с построением градуировочной сетки для эффективных микроскопических параметров: работы выхода, площади эмиссии и коэффициента усиления поля. С его применением было изучено поведение нанокомпозитного эмиттера на основе графена при изменении уровня напряжения. Проанализированы шумовые характеристики и их взаимосвязь с адсорбционно-десорбционными процессами.

DOI: $10.21883 /$ JTF.2017.07.44683.1952

\section{Введение}

Полевые квантовые эффекты - один из неотъемлемых атрибутов ныне интенсивно развивающейся наноэлектроники. Один из таких эффектов - полевая эмиссия электронов из многоострийных наноструктур. Этот вид эмиттеров уже зарекомендовал себя как энергетически выгодный и надежный источник электронов, однако комбинация сопутствующих эмиссии эффектов не позволяет использовать его в электронике, требуя тщательного изучения фундаментальных закономерностей самого процесса. Две важнейшие характеристики: стабильность эмиссионного тока и его зависимость от приложенного напряжения оказываются под влиянием ряда недоступных прямому контролю факторов: локальный джоулевый разогрев, адсорбционно-десорбционные процессы, ионная бомбардировка, воздействие пондеромоторных сил на морфологию поверхности, взаимное влияние эмитирующих наноцентров друг на друга, нелинейное изменение формы потенциального барьера с уровнем приложенного напряжения, а также зарядовые эффекты на поверхности эмиттера [1-4].

Один из предлагаемых ведущими учеными методов исследования поведения полевого эмиттера в различных режимах работы является так называемый SK-анализ. Для его проведения строится вольт-амперная характеристика (или серия характеристик) в координатах Фаулера-Нордгейма (ВАХ-ФН), производится ее аппроксимация прямолинейной зависимостью и полученные параметры этой зависимости (наклон $b$ и отсечка $a$ ) откладываются на SK-диаграмме (это диаграмма в координатах „Katamuki-Seppen“, т. е. „Slope-Intercept“ или „Наклон-Отсечка“).

Анализ полученного таким образом графика представляет собой довольно сложную теоретическую задачу. Основной идеей этого метода представляется создание градуировочной сетки, по которой можно определять микроскопические параметры эмиттера в ходе его работы: работу выхода, коэффициент усиления поля, средний радиус кривизны наноострий и общую площадь эмиссии. Впрочем, для расчета градуировочных „эквипотенциальных“ линий необходимо знать форму взаимосвязи этих параметров.

Изменение работы выхода электронов с концентрацией и типом адсорбировавшихся на поверхность эмиттера атомов еще в 1961 г. описал R. Gomer в своей книге „Field emission and field ionization“ [5].

Приращение величины работы выхода $\Delta \phi$ было оценено им так

$$
\Delta \phi=4 \pi N \alpha_{p} F,
$$

где $N-$ концентрация адатомов, $\alpha_{p}-$ коэффициент их поляризации, $F$ - локальное электрическое поле. Причем утверждалось, что электроотрицательные адатомы на поверхности эмиттера отталкивают электроны, за счет чего общий ток эмиссии уменьшается, а положительные - выступают в роли „окна“ в потенциальном барьере, за счет которого ток увеличивается.

Введение в стандартное уравнение Фаулера-Нордгейма зависимости (1) и разложение подэкспоненциального выражения в ряд Тейлора по $\Delta \phi$ позволило получить поправочный множитель к площади эмиссии, которым должна отличаться реальная площадь $S_{0}$ (площадь эмиссии без адатомов) от расчетной площади $S_{e}$ (рассчитанной без учета сорбционной поправки, но в присутствии адатомов):

$$
S_{e}=S_{0} \exp \left(-\left(\frac{3}{2}\right)(6.8) \cdot 10^{7} \alpha \gamma \phi^{1 / 2}\right),
$$

где $\gamma=4 \pi N \alpha_{p}$ - коэффициент пропорциональности $\Delta \phi$ и $F$ из формулы (1), а коэффициент $\alpha$ связан с отклонением формы потенциального барьера от треугольной под воздействием сил изображения (Nordheim, 1928 [6]). 
В 1976 г. Spindt использовал эту теорию, чтобы объяснить флуктуации эмиссионного тока из молибденового острия и перемещение эмитирующих областей на картине полевого эмиссионного проектора [7]. Коэффициент $\alpha$ был принят им равным 0.95. Тем же значением пользовались впоследствии и другие ученые.

В 1993 г. японские ученые Gotoh, Ishikawa и Tsuji впервые попробовали построить градуировочную сетку для SK-диаграммы на базе уравнения Фаулера-Нордгейма без сорбционных поправок [8]. Оценка работы выхода электронов из золотого микроострия с помощью этой сетки показала сомнительные результаты, поэтому было решено использовать поправку к работе выхода (1). Однако при этом в коэффициент изменения площади эмиссии был включен еще один фактор - коэффициент конверсии $\beta$ (определенный как $E / U$, где $U-$ внешнее напряжение, $E$ - поле на острие иглы):

$$
\begin{gathered}
S_{e}=S_{0} \exp \left(-9.74 \cdot 10^{7} \gamma^{\prime} \phi^{1 / 2}\right), \\
\gamma^{\prime}=\gamma \beta .
\end{gathered}
$$

Для определения неизвестных параметров $S_{0}$ и $\gamma$ использовались уравнения отсечки $a$ и наклона $b$ теоретической ВАХ-ФН и экспериментальные данные для золотого острия $(\phi=5 \mathrm{eV})$. В результате искомые параметры оказались равны: $S_{0}=10^{-8} \mathrm{~cm}^{2}, \gamma=10^{-13}$. На этот раз экспериментальные данные для других материалов (никель, молибден и хром), согласно градуировочной сетке на SK-диаграмме, показали правдоподобные результаты.

В 1996 г. та же группа ученых снова использовала зависимость (3) для построения градуировочной сетки по экспериментальным данным эмиссии из вольфрамовой проволоки с никелевым покрытием [9]. Авторы обстоятельно показали, что уменьшение/увеличение работы выхода приводит к смещению параметров BAX-ФН на SK-диаграмме вправо-вверх/влево-вниз, а уменьшение/увеличение радиуса кривизны эмитирующего острия - к смещению влево-вверх/вправо-вниз.

В 2004г. Gotoh, Ishikawa и Tsuji рассмотрели влияние на эмиссию платинового микроострия выдержки в атмосфере кислорода, водорода и углекислого газа [10]. На картинах полевого эмиссионного проектора наблюдалось блуждание пятен, которое авторы объяснили адсорбцией и десорбцией отдельных атомов в разных областях острия. Было показано, что выдержка эмиттера в водороде уменьшает эмиссионный ток (при этом уменьшается и относительный фликкерный шум), а в кислороде - уменьшает его еще сильнее. Выдержка в углекислом газе, наоборот, увеличивает уровень тока (при этом шум увеличился).

SK-диаграмма шумовой зависимости BAX показала линейную связь между наклоном и отсечкой:

$$
b=a A_{c}+B_{c} .
$$

Наблюдаемая линия была перпендикулярна линии изменения работы выхода на градуировочной сетке, что позволило утверждать, что шумовые флуктуации обусловлены изменением коэффициента усиления поля. Математический анализ этой линейной связи указал на то, что все шумовые ВАХ-ФН должны сходиться в одной точке с координатами $\left(1 / V_{c} \lg \left(I_{c} / V_{c}^{2}\right)\right)$ :

$$
\begin{gathered}
V_{c}=-A_{c}, \\
\lg \left(I_{c}=V_{c}^{2}\right)=-B_{c} / A_{c} .
\end{gathered}
$$

Экспериментально было показано, что точка эта с ростом напряжения линейно смещается, то есть наклон шумовой зависимости на SK-диаграмме линейно растет.

В работе [11] теми же учеными был проведен SK-анализ шумов тока эмиссии вольфрамового острия с использованием полевого эмиссионного проектора для визуализации работы наноцентров. Подаваемое на эмиттер напряжение было синусоидальным, а система регистрации компьютеризированной, что позволило получить большое количество экспериментальных ВАХ и соответственно высокую плотность точек на SK-диаграмме. Изменение амплитуды „сканирующего“ напряжения привело к смещению ВAX-ФН на SK-диаграмме и изменению расчетного коэффициента усиления поля.

Применение SK-анализа к массивам нанотрубок (толщиной $10 \mathrm{~nm}$, выращенных также на ITО-покрытии), позволило ученым из Южной Кореи оценить изменение микроскопических параметров эмиттера после его работы в режимах постоянного (DC) и переменного (AC) напряжения [12]. В режиме DC слегка изменилась работа выхода, а в режиме $\mathrm{AC}$ - сильно упал коэффициент усиления поля, что авторы объяснили увеличением радиуса концов нанотрубок (с 5 до $9.5 \mathrm{~nm}$ ) за счет десорбции адатомов.

Китайские ученые также воспользовались SK-диаграммой для анализа изменения многоострийного эмиттера из нанотрубок, выращенных CVD-методом на кремниевой подложке [13]. Эмиттер был подвергнут обработке кислородом с давлением $10^{-2} \mathrm{~Pa}$ в течение $2 \mathrm{~h}$ в ходе полевой эмиссии с уровнем тока $1.5 \mathrm{~mA}$. Наблюдалось падение коэффициента усиления поля с 5000 до 2000, что было связано авторами с выгоранием длинных нанотрубок под воздействием бомбардировки ионами атомов кислорода. При этом эмиссионный проектор показал существенное увеличение равномерности тока эмиссии по поверхности образца.

В 2013 г. американский ученый A. Persaud смоделировал шумовую зависимость ВАХ-ФН на SК-диаграмме посредством введения заданного распределения виртуальных наноцентров по высотам, радиусам и работам выхода [14]. Он показал, что смещение стохастического облака на SK-диаграмме налево и вниз может быть вызвано изменением не только работы выхода, но и количества эмитирующих наноцентров.

В 2014г. мы использовали его предположение о нормальности распределения наноцентров в нанокомпозитном эмиттере (нанотрубки в диэлектрической полимерной матрице) для анализа зависимости разброса 
наноцентров по высотам от уровня приложенного напряжения [15].

В настоящей работе представлено исследование эмиссионных свойств нанокомпозитного эмиттера на основе графена с применением SK-анализа по выше рассмотренным методикам.

\section{Исследовательский комплекс и программная обработка данных}

Экспериментальная установка по изучению эмиссионных свойств полевых эмиттеров подробно описана нами в [16]. Установка компьютеризирована и позволяет обрабатывать регистрируемые BAX и получать микроскопические параметры эмиттера в режиме онлайн. Программа обработки данных создана на платформе LabView 2015. Вольт-амперная характеристика регистрируются в „быстром“ режиме с частотой $50 \mathrm{~Hz}$ методом сканирования полусинусоидальными импульсами с управляемой амплитудой $U_{\max }$. Вакуум в камере поддерживается на уровне $10^{-7}$ Torr. Расстояние между электродами в настоящей работе составляло $d=300 \mu \mathrm{m}$.

Образцом для исследования был нанокомпозитный эмиттер, изготовленный на основе графена и полистирола. Подложкой служила круглая пластинка из нержавеющей стали (площадью $S_{k}=0.79 \mathrm{~cm}^{2}$ ). На нее методом вращающегося столика была нанесена суспензия: графен с полистиролом в соотношении 1/10 в органическом растворителе ортоксилоле. Для уменьшения размеров агломератов нанотрубок и лучшего растворения полимера суспензия перед нанесением в течение нескольких часов обрабатывалась в ультразвуковой ванне.

Онлайн анализ регистрируемых ВАХ использует уравнение полевой эмиссии, записанное в координатах Фаулера-Нордгейма:

$$
\lg \frac{j}{E_{0}^{2}}=\lg \left(A_{0}\right)+B_{\phi} \frac{1}{E_{0}},
$$

где $j-$ плотность эмиссионного тока, $E_{0}-$ напряженность электрического поля в области острия эмиттера, $A_{\phi}$ и $B_{\phi}-$ коэффициенты в приближении Елинсона [17], зависящие от $\phi-$ работы выхода эмиттера без адатомов (влияние адатомов на эмиссионный ток будет учтено в $\left.S_{e}\right)$ :

$$
\begin{gathered}
A_{\phi}=\frac{1.4 \cdot 10^{-6}}{\phi} \cdot 10^{4.39 \sqrt{\phi},} \\
B_{\phi}=-2.82 \cdot 10^{7} \phi^{3 / 2} .
\end{gathered}
$$

Или, выражая микроскопические величины $j$ и $E_{0}$ через макроскопические:

$$
\lg \frac{J}{E^{2}}=\lg \left(\frac{\beta^{2} A_{\phi} S_{e}}{S_{k}}\right)+\frac{B_{\phi}}{\beta} \frac{1}{E}=a+\frac{b}{E},
$$

где $J$ - макроскопическая плотность тока: $J=I / S_{k}=$ $=j S_{e} / S_{k}, E-$ макроскопическое поле, $\beta-$ коэффициент усиления поля: $E=E_{0} / \beta=U / d$.

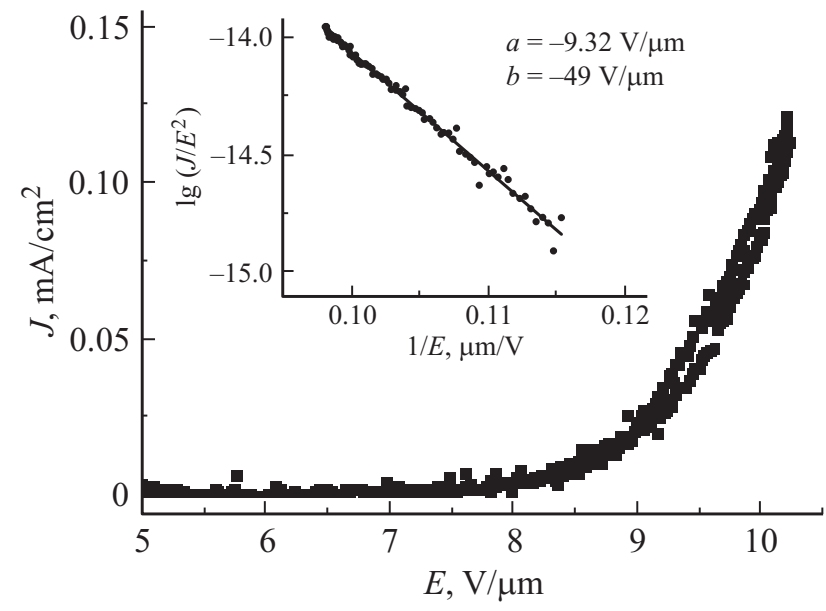

Рис. 1. Примеры зависимостей ВАХ и ВАХ-ФН (см. вставку), полученных в ходе эксперимента с графеновым нанокомпозитом.

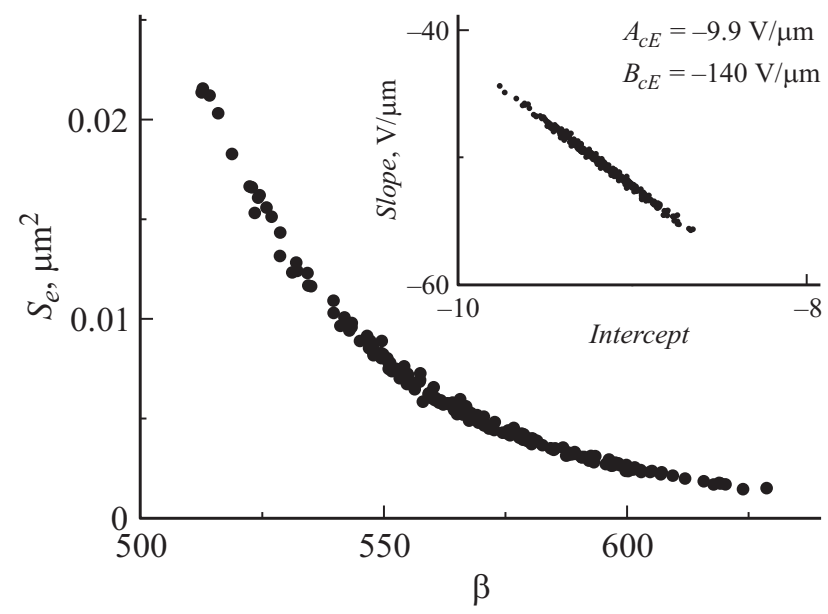

Рис. 2. Зависимость площади эмиссии $S_{e}$ от коэффициента усиления поля $\beta$, рассчитанная для шумовой SK-диаграммы (см. вставку) в ходе эксперимента.

Линейная аппроксимация экспериментальных данных, отложенных в тех же координатах, позволяет найти наклон $b$ и отсечку $a$ (рис. 1).

В ходе работы эмиттера его ВАХ демонстрирует фликкерный шум, так что наклон и отсечка у соответствующей аппроксимации имеют некоторый разброс. Полученный за несколько секунд работы эмиттера набор $b$ и $a$ откладывался на SK-диаграмме. Эта шумовая зависимость имеет линейный вид и может быть проанализирована в режиме онлайн с получением соответствующих коэффициентов $A_{c E}$ и $B_{c E}$ (аналогичных коэффициентам $A_{c}$ и $B_{c}$, введенным в (4), только для BAX в координатах $J$ и $E$ ).

Коэффициенты $a$ и $b$ связаны с параметрами $S_{e}, \beta$ и $\phi$ зависимостью (8):

$$
\begin{gathered}
a=\operatorname{gl}\left(\beta^{2} A_{\phi} S_{e} / S_{k}\right), \\
b=B_{\phi} / \beta .
\end{gathered}
$$




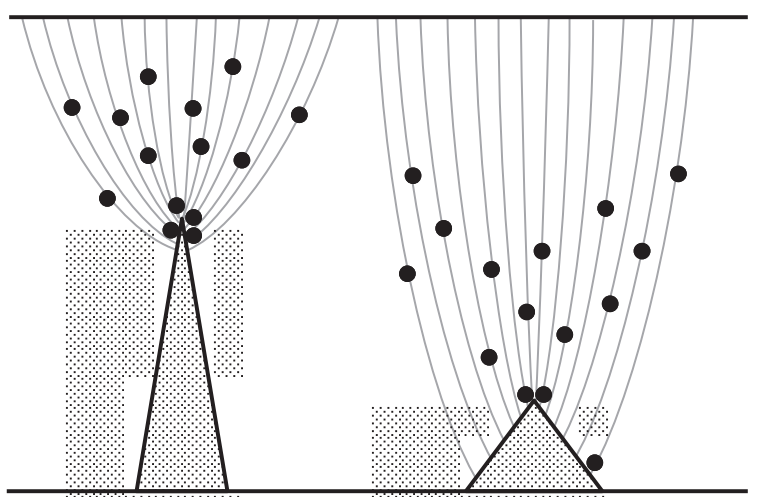

Pис. 3. Демонстрация эффекта фокусировки электрического поля остриями с разным коэффициентом усиления поля $\beta$ в области эмитирующей электроны вершины. Величина потока прилетающих на острие ионов пропорциональна плотности входящих в него силовых линий, которая задается формфактором $\beta$.

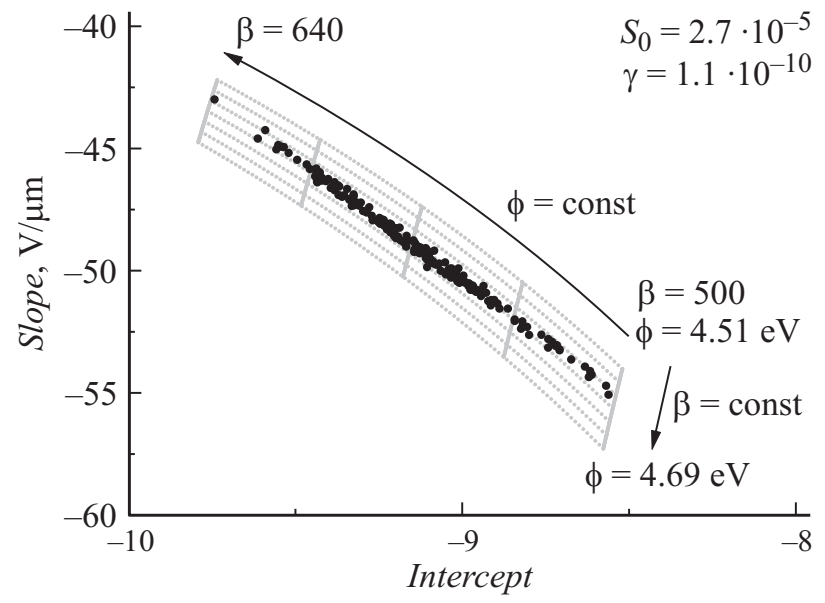

Рис. 4. Градуировочная сетка на SK-диаграмме, построенная в ходе эксперимента с коэффициентами $S_{0}$ и $\gamma$, определенными из зависимости $S_{e}(\beta)$.

Обрабатывающая сигнал программа вычисляет параметры $\beta$ и $S_{e}$ из экспериментальных величин $a$ и $b$ автоматически. Работа выхода $\phi$ в этом расчете задается экспериментатором (для исследуемого графенового нанокомпозита при малых эмиссионных токах мы принимали работу выхода $\phi=4.6 \mathrm{eV})$.

На рис. 2 представлена зависимость $S_{e}$ от $\beta$, которая получается в результате обработки данных из шумового облака. Ее форма близка к экспоненциальной, что позволяет нам применить аппроксимацию в согласии с зависимостью (3).

Отметим, что использование в (3) коэффициента усиления поля $\beta$ мы объясняем тем, что поток из межэлектродного пространства адсорбирующихся на каждый наноцентр атомов (определяющий в итоге среднее число адатомов $N$ на наноцентрах) должен быть пропорционален плотности приходящих к нему силовых линий, которая непосредственно зависит от $\beta$ (рис. 3 ).
В результате аппроксимации шумовых данных получаются адсорбционные коэффициенты $S_{0}$ и $\gamma$. С помощью этих коэффициентов отдельный модуль программы рассчитывает градуировочную сетку и накладывает ее на экспериментальную SK-диаграмму текущего BAX-ФH (рис. 4).

Тот факт, что проходящая через экспериментальную зависимость $b(a)$ эквипотенциальная линия сетки (с $\phi=4.6 \mathrm{eV}$ и варьируемым $\beta$ ) не имеет соответствующей прямолинейной формы, указывает на то, что (3) является всего лишь приближением, учитывающим только адсорбционную часть изменения измеряемой площади $S_{e}$. Другие влияющие на площадь факторы и правомерность применения градуировочной сетки мы обсудим в следующем разделе.

\section{Эксперимент с графеновым нанокомпозитом}

С целью изучения влияния уровня эмиссионного тока на микроскопические параметры эмиттера с помощью SK-анализа мы провели серию экспериментов, в которых эмиссионный ток постепенно (ступенчато) увеличивался и уменьшался. Результаты наложения градуировочной сетки на SK-диаграмму проведенного эксперимента представлены на рис. 5 (для удобства анализа на диаграмме оставлены только данные, соответствующие стабильному режиму работы эмиттера при разных уровнях тока). Градуировочная сетка была построена для первого флуктуационного облака при уровне тока $100 \mu \mathrm{A}$ (рис. 4).

Согласно полученной картине, с увеличением уровня тока работа выхода образца радикально увеличивается с 4.6 до $5.2 \mathrm{eV}$. Исходя из теоретического обоснования построения градуировочной сетки, это смещение вызвано изменением равновесной концентрации адатомов на поверхности эмиттера $N$. Концентрация эта зависит от скоростей адсорбционно-десорбционных процессов, которые меняются с ростом амплитуды сканирующего напряжения. Вызвано это может быть изменением величины пондеромоторных сил, срывающих адатомы с наноцентров, изменением локальной температуры наноцентров под воздействием джоулевого тепла, изменением колебательных условий упругих листов графена, а также изменением плотности потока ионов при изменении локальной фокусировки полей (рис. 3).

Вместе с ростом работы выхода на полученной SKдиаграмме падает расчетный коэффициент усиления поля $\beta$ с 550 до 460. Уменьшение общего $\beta$ должно быть связано с включением в процесс эмиссии более „низких“ наноцентров, что подтверждается проведенными нами опытами на аналогичных нанокомпозитах с использованием эмиссионного проектора [16].

Судя по наличию гистерезиса на SK-диаграмме, изменение адсорбционно-десорбционных процессов при уменьшении тока отличается от того же изменения при его увеличении. Это может быть связано с джоулевым 

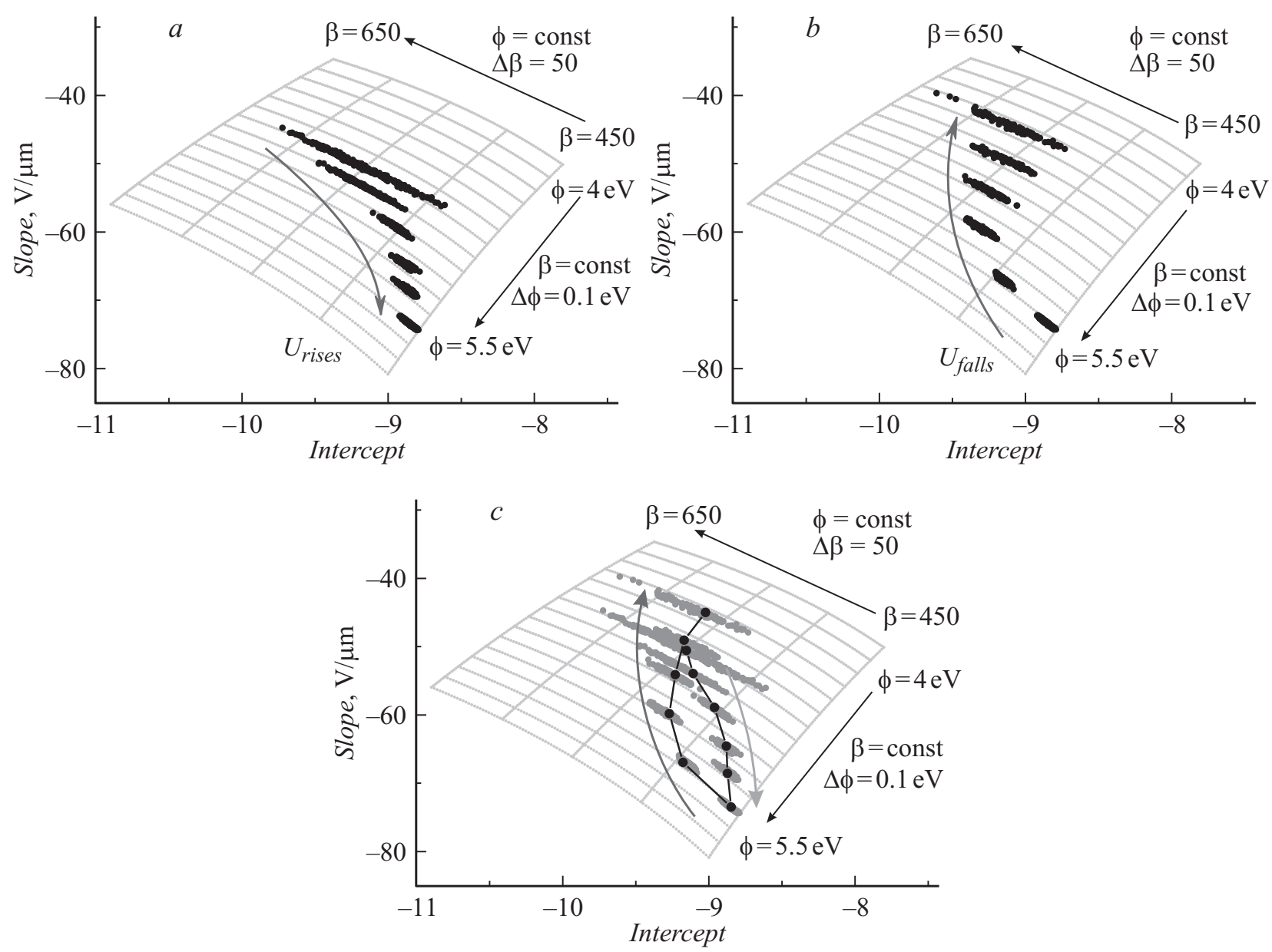

Рис. 5. SК-диаграммы изменения параметров ВАХ-ФН нанокомпозита „графен/полистирол“ при ступенчатом увеличении (a) и уменьшении $(b)$ эмиссионного тока. Градуировочная сетка построена для первого флуктуационного облака при уровне тока $100 \mu \mathrm{A}$. На рис. 5, с представлен гистерезис центров шумовых облаков, наблюдаемый в эксперименте.

разогревом наноцентров и изменением их токовой нагрузки, что отмечалось нами в экспериментах с другими нанокомпозитами, где наблюдался подобный гистерезис [18].

Рассмотрим относительное расположение флуктуационных облаков и градуировочных линий на SK-диаграмме. Облака имеют почти линейную форму и располагаются по касательной к эквипотенциальным кривым с соответствующими работами выхода. Это указывает на принципиальное различие двух процессов: шумовое изменение эмиссионного тока при постоянном уровне амплитуды сканирующего напряжения и изменение тока при значительном изменении $U_{\max }$.

Первый процесс, очевидно, связан с блужданиями эмиттирующих областей по поверхности образца (мы наблюдали их с помощью эмиссионного проектора [19]). Многие авторы связывают эти блуждания со стохастикой адсорбционных процессов. Подобная стохастика должна влиять на общий коэффициент усиления поля $\beta$, так как, адсорбируясь случайным образом, адатомы „включают“ в эмиссию то высокие наноцентры, то низкие. В то же время возле своих наиболее вероятных значений будут флуктуировать и общая работа выхода эмиттера $\phi$, зависящая от среднего числа адатомов на один наноцентр, и общая площадь эмиссии $S_{0}$, зависящая от числа „включившихся“ наноцентров. Поэтому флуктуационная зависимость на SK-диаграмме имеет вид наклонного эллипса.

Второй же процесс кардинально меняет условие флуктуационного баланса, приводя к изменению скорости адсобционно-десорбционных процессов, т.е. меняет наиболее вероятные значения определяющих ток параметров: $\beta, \phi$ и $S_{0}$. Причем изменение работы выхода будет в данном случае ключевым.

В обоих процессах фигурирует изменение коэффициента усиления поля $\beta$, которое должно приводить к изменению работы выхода по (3). Однако предложенный нами механизм изменения общей работы выхода эмиттера за счет изменения плотности потока ионизированных адатомов к остриям (рис. 3) может быть достаточно медленным, чтобы не успевать за флуктуационными скачками $\beta$. Так что шумовая зависимость ВАХ-ФН на SK-диаграмме может быть не подвержена соответствующим изменениям $\phi$. 


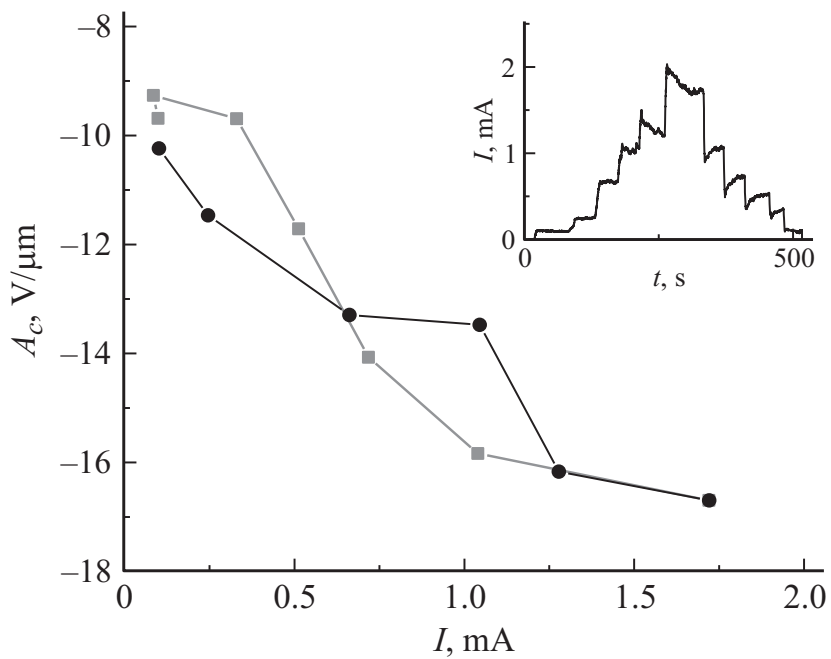

Рис. 6. Зависимость наклона стохастического облака ВАХ-ФН на SK-диаграмме от уровня эмиссионного тока (только для роста тока). Изменение тока со временем показано на вставке.

В принципе линейность шумовых облаков на SKдиаграмме можно получить математически из (8), если принять $J$ и $E$ постоянными:

$$
a=\left(\lg \frac{J}{E^{2}}\right)-\left(\frac{1}{E}\right) b .
$$

Тогда из уравнений (9) и (10) вытекает взаимосвязь микроскопических $S_{e}$ и $\beta$, математически задающая линейную форму стохастического облака:

$$
S_{e}=\frac{J}{E^{2}}\left(\frac{S_{k}}{\beta^{2} A_{\phi}}\right) \cdot 10^{\frac{-B_{\phi}}{E \beta}} .
$$

Единственной точкой, где $J$ и $E$ постоянны для всех стохастических $a$ и $b$, является точка пересечения соответствующих им BAX (5) с координатами $\left(E_{c}, J_{c}\right)$. Исходя из оценок, проведенных нами на базе экспериментальных данных, напряженность поля в этой точке составляет $E_{c \text { loc }} \approx 5 \cdot 10^{7} \mathrm{~V} / \mathrm{cm}$ (для уровня тока $100 \mu \mathrm{A}$ из рис. 4 получаем $A_{c E} \approx-10 \mathrm{~V} / \mu \mathrm{m}$, откуда $E_{c} \approx 10 \mathrm{~V} / \mu \mathrm{m}$, что при учете коэффициента усиления поля $\beta \approx 500$ дает локальное поле $\left.E_{c \text { loc }} \approx 5 \cdot 10^{7} \mathrm{~V} / \mathrm{cm}\right)$. Это достаточно высокие поля, близкие к напряженности снятия потенциального барьера $\left(E_{c} \sim 10^{8} \mathrm{~V} / \mathrm{cm}\right.$ для $\phi=4 \mathrm{eV}[20])$.

Действующие на адатомы пондеромоторные силы при таких полях могут заставлять их десорбироваться, что существенно сближает стохастические характеристики друг к другу и заставляет ВАХ пересекаться в одной точке.

Увеличение же амплитуды напряжения меняет адсорбционно-десорбционный баланс и заставляет десорбироваться большее количество атомов при максимальном поле в импульсе $E_{c}$, за счет чего точка пересечения BAX смещается и соответственно меняется наклон флуктуационного облака $A_{c E}$ (рис. 6). То есть ВАХФН образца в режиме стабильного тока эмиссии стохастически „вращаются“ вокруг точки, соответствующей минимальному количеству адатомов на эмиттере при заданной амплитуде напряженности поля.

\section{Заключение}

Мы разработали методику онлайн анализа SKдиаграмм вольт-амперных характеристик полевых эмиттеров большой площади. Градуировочная сетка, построенная по шумовой зависимости расчетной площади эмиссии от эффективного коэффициента усиления поля, позволила проанализировать изменение микроскопических параметров эмиттера, созданного на основе нанокомпозита графен/полистирол, в ходе последовательного увеличения и уменьшения амплитуды сканирующего напряжения.

Адекватность построения градуировочной сетки по шумовым зависимостям была принята на основании утверждения, что оба процесса - шумовые вариации площади эмиссии с коэффициентом усиления поля и закономерное изменение этих же параметров при изменении уровня сканирующего напряжения - являются проявлением суть одних и тех же стохастических адсорбционно-десорбционных процессов, отличающихся лишь скоростью изменения равновесной работы выхода образца.

Наблюдаемый на диаграмме гистерезис ВАХ мы связываем с джоулевым разогревом наноцентров и изменением их токовой нагрузки. Вариации работы выхода объясняются изменением равновесной концентрации адатомов на эмитирующих наноцентрах, а вариации коэффициента усиления поля - включением в процесс эмиссии наноцентров с более низкими эффективными высотами.

Дальнейшее изучение закономерностей в полевых эмиссионных процессах многоострийных эмиттеров c применением SK-анализа будет продолжено нами с применением полевого эмиссионного компьютеризированного проектора, а также моделированием многоострийных эмисионных систем с адсорбционнодесорбционными стохастическими процессами.

\section{Список литературы}

[1] Елецкий А.В. // УФН. 2010. Т. 180. Вып. 9. С. 897-930. J. Success. Phys. sci. 2010. Vol. 180. N 9. P. 897.

[2] Васильева Е.А., Клещ, В.И., Образцов А.Н. // ЖТФ. 2012. Т. 82. Вып. 7. С. 107-111.

[3] Forbes R.G. // Nanotechnology. 2012. Vol. 23. P. 1-095706-12.

[4] Yue S.L., Gu C.Z., Shi C.Y., Zhi C.Y. // Appl. Surf. Sci. 2005. Vol. 251. P. 219.

[5] Gomer R. Field emission and field ionization. Cambridge: Harvard Uneversity Press, 1961. 195 p.

[6] Nordheim L.W. // Proc. Roy. Soc. Lond. 1928. Vol. 121. N 788. P. 639. 
[7] Spindt C.A., Brodie I., Humphrey L., Westerberg E.R. // J. Appl. Phys. 1976. Vol. 47. P. 5263.

[8] Ishikawa J., Tsuji H., Gotoh Y., Inoue K., Nagao M., Sasaki T., Kaneko T.J. // Jpn. J. Appl. Phys. 1993. Vol. 32. Part 2. P. L342-L345.

[9] Gotoh Y., Nagao M., Matsubara M., Inoue K., Tsuji H., Ishikawa J. // Jpn. J. Appl. Phys. 1996. Vol. 35. P. L1297L1300.

[10] Gotoh Y., Nagao M., Nozaki D., Utsumi K. // J. Appl. Phys. 2004. Vol. 95. P. 1548.

[11] Kawasaki M., He Z., Gotoh Y., Tsuji H., Ishikawa J. // J. Vac. Sci. Technol. B. 2010. Vol. 28. P. C2A82.

[12] Baik C.W., Lee J., Choi J.H., Jung I., Choi H.R., Jin Y.W. // Appl. Phys. Lett. 2010. Vol. 96. P. 1-023105-3.

[13] Zhang Y., Liao M.X., Deng S.Z., Chen J. // Carbon. 2011. Vol. 49. P. 3299-3306.

[14] Persaud A.J. // Appl. Phys. 2013. Vol. 114. P. 1-154301-6.

[15] Kolosko A.G., Popov E.O., Filippov S.V., Romanov P.A. // J. Vac. Sci. Technol. B. 2015. Vol. 33. P. 03C104-6.

[16] Попов Е.О., Колосько А.Г., Филиппов С.В., Романов П.А., Федичкин И.Л. // Журнал „Наноматериалы и наноструктуры - XXI век“. 2016. Вып. 1. С. 14-26.

[17] Елинсон М.И. Ненакаливаемые катоды. М.: Сов. радио, 1974. $336 \mathrm{c}$

[18] Колосько А.Г., Попов Е.О., Филиппов С.В., Романов П.А. // ПЖТФ. 2014. Т. 40. Вып. 10. С. 65-72.

[19] Kolosko A.G., Popov E.O., Filippov S.V., Romanov P.A., Terukov E.I. // IEEE $28^{\text {th }}$ Intern. Vacuum Nanoelectron. Conf. Guanggzhou, China, 2015. P. 40.

[20] Елинсон М.И., Васильев Г.Ф. Автоэлектронная эмиссия. М.: Гостехиздат. 1958. 272 с. 